\title{
Experimental Measurements of Regional Lung Deposition in Taiwanese
}

\author{
Chih-Wei Lin ${ }^{1}$, Sheng-Hsiu Huang, ${ }^{1}$, Kuang-Nan Chang ${ }^{1}$, Yu-Mei Kuo ${ }^{2}$, Huey-Dong Wu ${ }^{3}$, \\ Chane-Yu Lai ${ }^{4}$, Chih-Chieh Chen ${ }^{1 *}$
}

\author{
${ }^{1}$ Graduate Institute of Occupational Medicine and Industrial Hygiene, College of Public Health, National Taiwan \\ University, Taipei 10055, Taiwan \\ ${ }^{2}$ Department of Occupational Safety and Health, Chung Hwa University of Medical Technology, Tainan 71703, Taiwan \\ ${ }^{3}$ Department of Integrated Diagnostics and Therapeutics, National Taiwan University Hospital, Taipei 10051, Taiwan \\ ${ }^{4}$ Department of Occupational Safety and Health, Chung Shan Medical University, Taichung 40201, Taiwan
}

\begin{abstract}
Inhalation is the most important route of entry for aerosol particles. Deposition in the respiratory tract is affected by many factors, such as particle size, shape, charge, density, breathing pattern and physical tract structure. However, most of the currently available lung deposition data are on Caucasians, with very limited data on Taiwanese. Therefore, it is essential to clarify whether there is a significant difference in respiratory tract deposition between Caucasian and Taiwanese individuals. This work is thus aimed at characterizing regional lung deposition in Taiwanese.

A rapid method of measuring regional lung deposition was employed in the present study. The experimental system consisted of an aerosol chamber, a mouthpiece, a pneumotachograph flow meter, and a particle counter. A cylinder-piston type breathing machine was used to generate a series of "standard" breathing patterns for subjects to follow. In addition to oral breathing, nasal breathing was also conducted. A special respirator was employed to facilitate measurements of the nasal route deposition. The regional lung deposition data obtained in this work showed good agreement with previous studies based on the bolus technique, indicating that the difference in lung deposition between Taiwanese and Caucasian individuals is negligible. The local deposition efficiency increased with the penetration volume. This increased trend was particularly prominent in the deep lungs, which was likely due to the dilution effect caused by the relatively clean air in the functional residual capacity. When a fixed fraction of the forced vital capacity was used to replace the fixed tidal volume, the total lung deposition became less dependent on the tidal volume. Primarily due to nostril hairs and the complex configuration of the nasal turbinate, the local deposition efficiency of the nasal route was higher than that of the oral route but only in the early stage of penetration $(200 \mathrm{~mL})$.
\end{abstract}

Keywords: Aerosol deposition; Lung function; Breathing pattern; Tidal volume.

\section{INTRODUCTION}

Inhalation is the most important route for aerosol particles in the atmosphere to enter the body. The index of lung function measurement and the biological response caused by these inhaled particles vary with their site of deposition in the respiratory tract and the physiological or toxic effects within the target tissue. Particles in the ambient air are associated with adverse health effects such as cough, irritation, or allergy. The health effect can be acute or chronic. It depends on the particle composition, size, and the region of lung, so it is necessary to determine the total

\footnotetext{
* Corresponding author.

Tel.: 886-2-33668086; Fax: 886-2-23938631

E-mail address: ccchen@ntu.edu.tw
}

and regional distribution of particle deposition within the human respiratory tract for a proper evaluation of health risks. In addition, inhalation is an efficient way to deliver pharmaceutical aerosols used to treat respiratory diseases. Particles deposit within respiratory system depending on particles' size distribution, shape, and charge condition (Cohen et al., 1998; Ali et al., 2009; Sturm and Hofmann, 2009; Darquenne, 2012). Furthermore, it depends on breathing conditions such as breathing frequency, pause and flow rate (Kim and Jaques, 2005; Kim and $\mathrm{Hu}, 2006$; Inthavong et al., 2010; Patterson et al., 2014).

Various methods have been applied to measure regional lung deposition. Previously, regional lung deposition has been measured by inhalation of particles labeled with $\gamma$-emitting radionuclide (Lippmann and Albert, 1969; Stahlhofen et al., 1980; Hashish et al., 1998; Möller et al., 2006; Rissler et al., 2017). However, subjects may be exposed to a high dosage of $\gamma$ radionuclide. The process is quite cumbersome 
and time-consuming. Hollow models (cast) of human respiratory tract were frequently used to measure regional lung deposition (Chan and Lippmann, 1980; Grgic et al., 2006; Su and Cheng, 2006; Ali et al., 2008; Zhou et al., 2011). However, casts are not exactly the same as the real human respiratory tract, so making a realistic cast is still very challenging, especially for the peripheral parts of the lung. Recently, the serial bolus delivery method has been commonly employed to measure regional lung deposition (Kim et al., 1996; Brand et al., 1997; Heyder et al., 1988). The method is non-invasive, and does not require radioactive labelling of aerosols. However, measuring longitudinal distribution of deposited particles in the lungs using the bolus technique is still tedious and time-consuming and a high concentration of challenge aerosols is needed to ensure accurate measurement.

Until now, most experimental studies examining human regional lung deposition were on Caucasians. Lung deposition data on Taiwanese are very limited. Therefore, a database is needed to clarify whether there is a significant difference in respiratory tract deposition between Caucasians and Taiwanese. This is an essential step from the perspective of occupational hygiene, since it may profoundly affect the occupational hygiene regulations and standards. Therefore, this work aimed to characterize the regional lung deposition of Taiwanese.

\section{METHODS}

The experimental apparatus for rapid measurement of regional lung deposition is shown in Fig. 1. To avoid hygroscopic growth of test aerosol in the respiratory tract, di(2-ethylhexyl) sebacate (DEHS) was chosen as the test agent. A condensation monodisperse aerosol generator
(CMAG; Model 3475, TSI Inc., St. Paul, MN, U.S.A.) was chosen to generate micrometer-sized monodisperse DEHS aerosols. A constant output atomizer (Model 3076, TSI Inc.) and an electrostatic classifier (Model 3080, TSI Inc.) were used to generate submicrometer-sized monodisperse particles. Two aerosol spectrometers were used to confirm the monodisperse size distribution and number concentration: an Aerodynamic Particle Sizer (APS; Model 3321, TSI Inc.) for particles larger than $0.7 \mu \mathrm{m}$, and scanning mobility particle sizer (SMPS; Model 3936, TSI Inc.) for particles smaller than $0.6 \mu \mathrm{m}$. Before introducing the particles into the mixing chamber, a $25 \mathrm{mCi}^{241} \mathrm{Am}$ radioactive source was used to neutralize the aerosol particles to the Boltzmann charge equilibrium. After the generated aerosols were introduced into the mixing chamber, a filtered dilution air flow of $100 \mathrm{~L} \mathrm{~min}^{-1}$ was employed to mix and carry the generated particles. A humidifier was employed to condition the filtered air to a relative humidity $(\mathrm{RH})$ of $70-80 \%$. This was for the comfort of the human subjects.

The main sampling train used in a previous study (Chang et al., 2013) was slightly modified to consist of a mouthpiece, a pneumotachograph flow meter (Fleisch $1 \mathrm{TH}$ ), and a particle counter. The mouthpiece was attached to the flow meter in line with a minimum dead space. In the present study, a condensation particle counter (CPC; Model 3025, TSI Inc.) was used to measure the particle number concentrations. During respiration, the aerosol was sampled continuously by diverting a stream of air from the sampling train into the CPC via the sidearm port attached to the mouthpiece. By using a PC-LabCard (PCI-1710HG-A, Advantech Inc., Taipei, Taiwan) and a personal computer, the CPC measured the particle number concentration at a frequency of $100 \mathrm{~Hz}$. All the air flows were controlled and monitored by mass flow controllers (Hasting Instruments, Hampton, VA, U.S.A.),

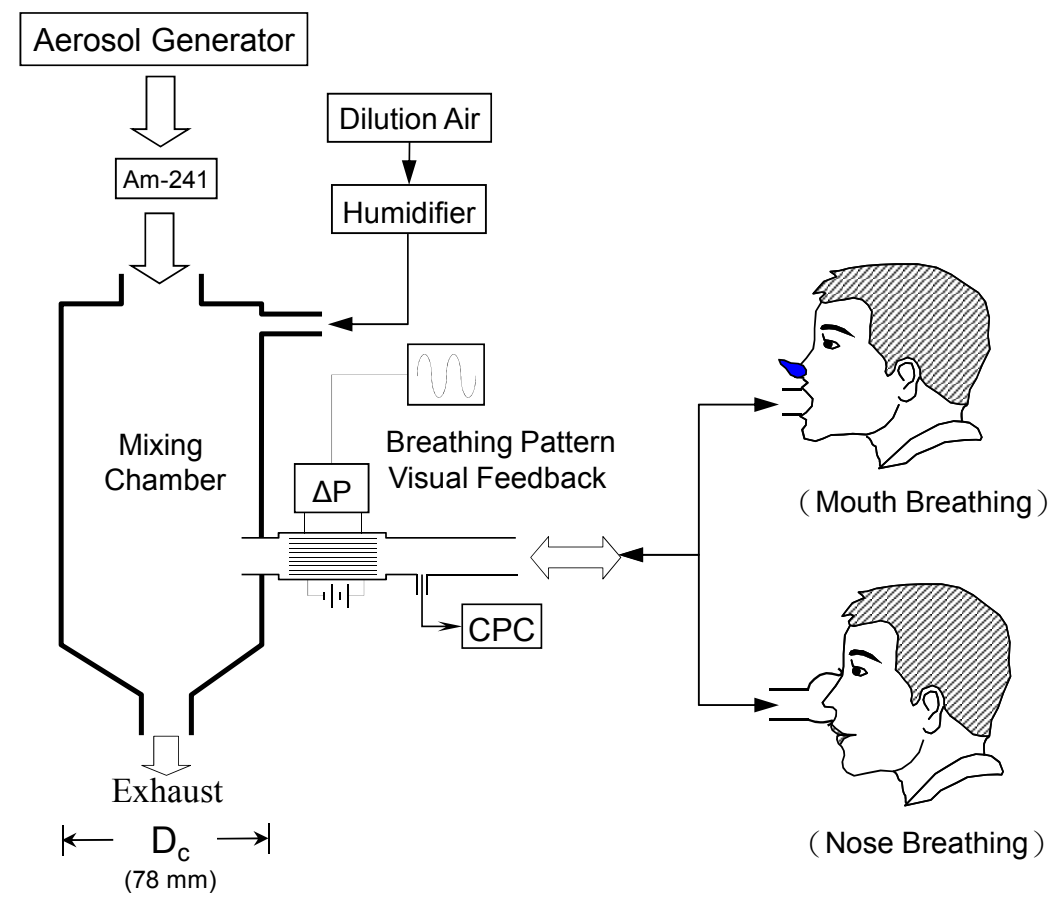

Fig. 1. Schematic diagram of the experimental system set-up. 
which were calibrated against an electronic bubble meter (Gilibrator, Gilian Instrument Crop., Wayne, NJ, U.S.A.) or a dry gas meter if the flow was higher than $30 \mathrm{~L} \mathrm{~min}^{-1}$.

A pressure transducer (PX653-01D5V, Omega Engineering Inc., Stamford, CT, U.S.A.) and PC-LabCard were connected to the flow meter to monitor the pressure change during breathing. Before the test, a homemade cylinder-piston breathing machine (cyclic flow model) was used to generate a series of "standard" breathing patterns, i.e., combinations of different tidal volumes and breathing frequencies. The subjects were instructed to practice and follow the breathing pattern shown on a monitor. Standard breathing patterns were plotted on the computer monitor placed in front of the subject for him/her to follow. It normally took subject more than 30 minutes to learn to trace the breathing patterns.

There were 10 male and 2 female subjects recruited in the present study. All subjects were asked to read and sign a consent form approved by National Taiwan University. Subject characteristics and lung function test results are shown in Table 1. The subjects could be described as young and healthy. Each lung deposition test included at least 15 breathing cycles. All tests were repeated at least 5 times per day and for more than 3 days. In addition to the fixed tidal volume of $500 \mathrm{~mL}$, the subjects were also asked to perform $20 \%$ of FVC (Forced Vital Capacity) to examine the effect on lung deposition. A high fraction of FVC was not suitable to perform because some subjects' high breathing flow might cause flow fluctuation of the particle counter.

To estimate the longitudinal distribution of aerosol deposition, tidal volume can be divided into infinitesimally small volume elements, or $n$ elements. Aerosol particles within each volume element of the respiratory tract system are assumed to have a regional deposition efficiency of $x_{i}$ as they are inhaled and exhaled again with the same deposition efficiency penetrating through the same volume element $(i)$ (Kim et al., 1996; Brand et al., 1999). Aerosol recovery from the $i^{\text {th }}$ volume element, $R C_{i}$, can be obtained by

$R C_{i}=\prod_{k=1}^{i}\left(1-x_{k}\right)^{2}$
Aerosol deposition fraction in $i^{\text {th }}$ volume element $\left(D F_{i}\right)$ is the sum of depositions during inspiration and expiration. The local deposition fraction in the $i^{\text {th }}$ volume element, $L D F_{i}$, can then be expressed as

$$
L D F_{i}=\frac{1}{n} \sum_{j=1}^{n} D F_{i}
$$

In addition to mouth breathing, nose breathing was also performed in the present study. A special respirator was employed to facilitate the nasal route deposition experiments. The space of a nose respirator together with the sample train was about $50 \mathrm{~cm}^{3}$, almost the same as the mouth route. Before nose breathing tests, the leakage test was performed to ensure the special respirator was well fitted for the subject.

\section{RESULTS AND DISCUSSION}

Twelve subjects completed lung deposition efficiency measurements for $1 \mu \mathrm{m}$ particles at a tidal volume of $500 \mathrm{~mL}$ and breathing frequency of 15 breaths $\mathrm{min}^{-1}$. Fig. 2 shows the local deposition efficiency curves for each $50 \mathrm{~mL}$ volumetric region as a function of penetration volume for all 12 subjects. The trend (circle symbol with solid line) of Taiwanese regional lung deposition was similar to Kim's data (Kim et al., 1996), showing that local deposition values increased with increasing penetration volume. However, the local deposition efficiency $(0-400 \mathrm{~mL})$ measured in the present study was slightly higher (about 1-3\%) than Kim's. Yet, the difference in local deposition efficiency increased sharply when entering the deep penetration volume (400$500 \mathrm{~mL}$ ). This mismatch with Kim's data was likely due to (1) the two experimental systems not being exactly the same: The bolus technique was used in Kim's study, while the rapid measurement system was employed in the present study; and (2) the breathing patterns differed: Constant velocity was adopted in Kim's study, while the sinusoidal pattern was used in this work. Furthermore, the difference in the deep lung was likely due to the dilution effect

Table 1. Subject characteristics and lung function test results.

\begin{tabular}{lllllllll}
\hline Subject & Gender & Age & Height, cm & Weight, Kg & FVC, L & FEV1, L & VT, L & 20\% FVC, L \\
\hline 1 & M & 29 & 160 & 62 & 3.37 & 2.79 & 0.65 & 0.67 \\
2 & M & 24 & 171 & 84 & 5.18 & 4.67 & 0.55 & 1.04 \\
3 & F & 23 & 158 & 48 & 3.11 & 2.74 & 0.45 & 0.62 \\
4 & M & 37 & 168 & 60 & 4.14 & 4.04 & 0.75 & 0.83 \\
5 & M & 23 & 172 & 60 & 4.6 & 3.72 & 0.73 & 0.92 \\
6 & M & 29 & 174 & 79 & 3.43 & 2.9 & 0.72 & 0.67 \\
7 & M & 33 & 175 & 68 & 4.64 & 3.95 & 0.82 & 0.93 \\
8 & M & 25 & 170 & 69 & 4.09 & 3.45 & 0.63 & 0.82 \\
9 & M & 24 & 170 & 60 & 4.27 & 3.84 & 0.64 & 0.85 \\
10 & M & 22 & 180 & 65 & 4.79 & 4.09 & 0.6 & 0.96 \\
11 & F & 22 & 156 & 50 & 2.91 & 2.47 & 0.67 & 0.58 \\
12 & M & 22 & 175 & 60 & 4.61 & 3.88 & 0.61 & 0.92 \\
\hline
\end{tabular}

FVC, forced vital capacity; FEV1, forced expiratory volume in $1 \mathrm{~s}$; VT, Tidal Volume. 


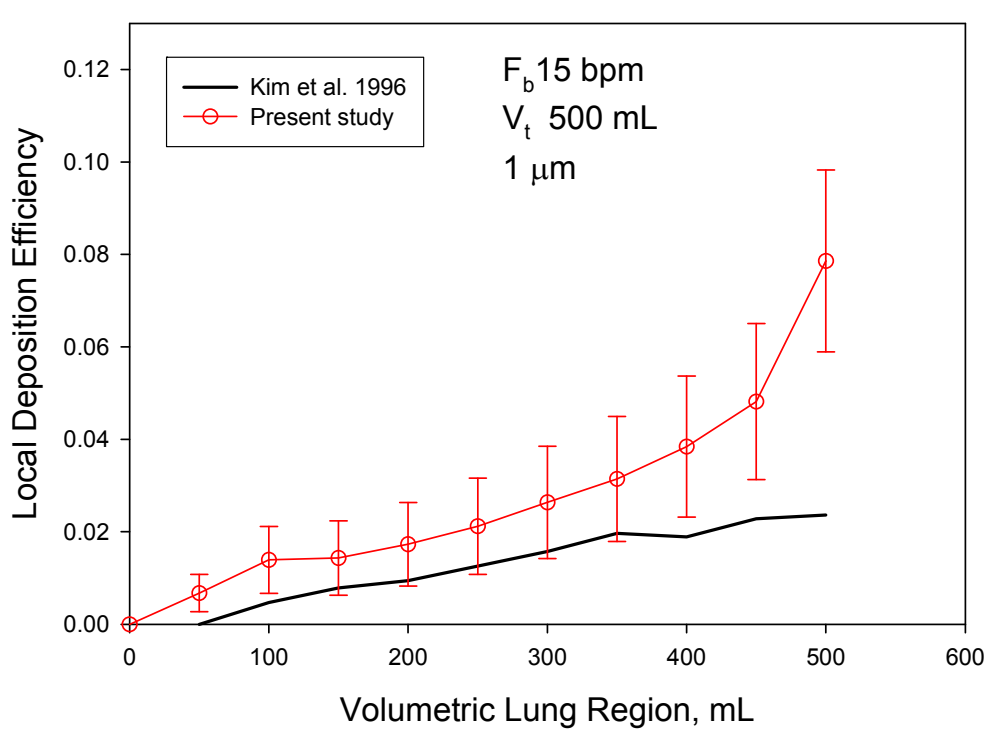

Fig. 2. Local deposition efficiency (for each $50 \mathrm{~mL}$ volumetric region) as a function of penetration volume compared with Kim's data.

caused by the clean air staying in the functional residual capacity (FRC). Note, aerosol particles remaining in the FRC were very likely deposited due to either diffusion (for small particles) or gravitational settling (for large particles) because of long retention time. Therefore, the FRC air could be regarded as aerosol-free to dilute the aerosol concentration entering the alveolar region.

Moreover, the regional lung deposition of each subject might vary quite significantly, as shown in Fig. 3. For example, Subject 7 had higher regional lung deposition than Subject 5 throughout all penetration volumes. These two subjects began to show different regional deposition efficiency values after the first $50 \mathrm{~mL}$, or the mouth region. This mismatch was particularly pronounced in the $300 \mathrm{~mL}$ of volumetric lung region, and the difference slightly shrank when going farther into the alveolar region. Both subjects were asked to perform the same breathing pattern of tidal volume $(500 \mathrm{~mL})$ and breathing frequency $\left(15\right.$ breaths $\left.\mathrm{min}^{-1}\right)$. Since both subjects shared about the same lung function and deposition efficiency curves were very repeatable, the difference in regional lung deposition was likely due to anatomical and physiological reasons, i.e., the dimensions of the respiratory tract and/or the thickness of the mucus layer.

The local deposition efficiency values of Subject 1 over 15 months are shown in Fig. 4. The tests were repeated at least 3 times each month. The results indicated the trend of local deposition efficiency was consistent and always increased with penetration volume. However, the local deposition efficiency values might vary with time but not in a particular order. The difference between the highest and lowest deposition efficiency curves was about a factor of 2 . The reasons for this inconsistency require further investigation.

The regional lung deposition efficiency data could be integrated to form total lung deposition efficiency data. In the present study, the total lung deposition for particles sized $20 \mathrm{~nm}-1 \mu \mathrm{m}$, at tidal volume of $500 \mathrm{~mL}$ and breathing frequency of 15 breaths $\min ^{-1}$ are shown in Fig. 5, to compare with Kim's (Jaques and Kim, 2000; Kim and Hu, 2006) and Heyder's data (Heyder et al., 1986). Both male and female data of Kim agreed quite well with the solid line which was the best fit of Heyder's data. In Fig. 5, the total deposition fraction decreased with increasing particle size from 20 to $300 \mathrm{~nm}$, apparently because of decreasing particle diffusivity. For particles larger than $300 \mathrm{~nm}$, the total deposition fraction then increased with particle size because of increasing inertial impaction. Overall, the deposition efficiency curve showed a "collection minimum" at $0.3 \mu \mathrm{m}$, an apparent characteristic of the mechanical filter. The deposition efficiency data collected in this work showed good agreement with previous studies, and although our data were slightly lower, the difference was not statistically significant.

In general, the data analysis of regional lung deposition in previous studies was all based on fixed tidal volume. However, lung function varied among voluntary subjects, as shown in Table 1. For example, the forced vital capacity ranged from $2.91 \mathrm{~L}$ (Subject 11) to $4.79 \mathrm{~L}$ (Subject 10), indicating people breathed differently in terms of tidal volume. Therefore, it was of interest to measure the lung deposition at $20 \% \mathrm{FVC}$, in addition to the fixed tidal volume of $500 \mathrm{~mL}$. Fig. 6 shows the total deposition of both fixed tidal volume of $500 \mathrm{~mL}$ and $20 \%$ FVC of 7 subjects, breathing $1 \mu \mathrm{m}$ particles at 15 breaths $\mathrm{min}^{-1}$. For fixed tidal volume, the deposition fraction varied, ranging 0.13-0.18. The deposition fraction of each subject was higher when performing $20 \% \mathrm{FVC}$, because the minute volume was higher than $500 \mathrm{~mL}$. Lung deposition increasing with tidal volume under the same breathing frequency has been well documented, because the aerosol particles had to travel a greater distance into the deep lung, resulting in higher aerosol deposition. However, the deposition efficiency of $20 \%$ FVC was clearly less dependent on tidal volume, indicating aerosol deposition efficiency was about the 


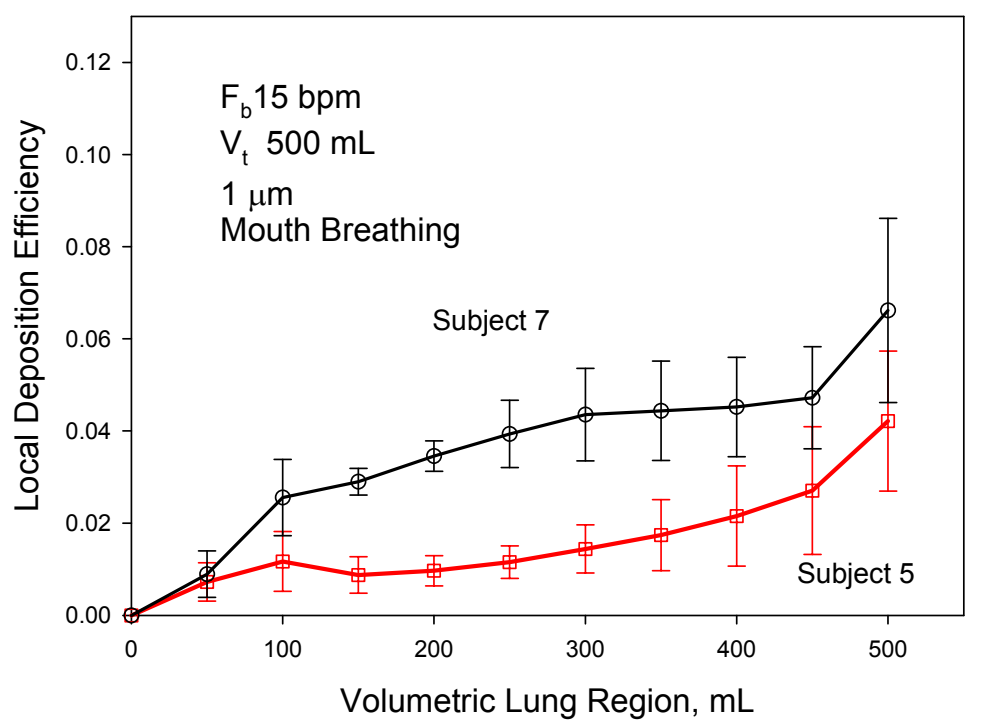

Fig. 3. Comparison of local deposition efficiency of Subject 5 and 7 for mouth breathing.

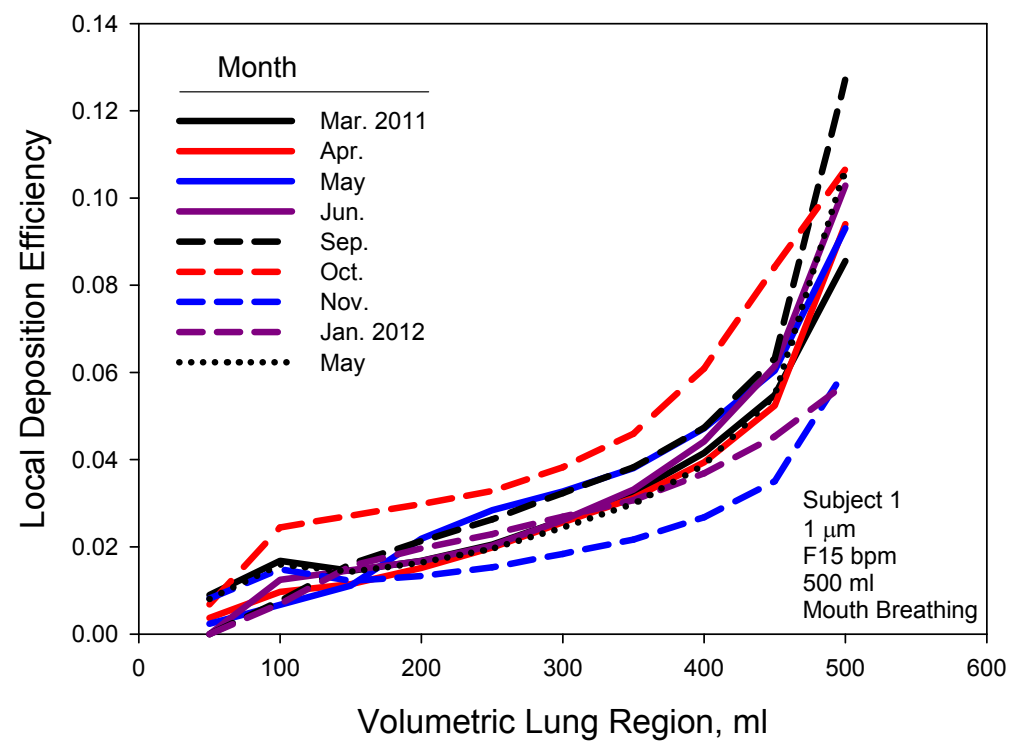

Fig. 4. Local deposition efficiency of Subject 1 in different months.

same for participating subjects. Nevertheless, the deposited aerosol mass increased with tidal volume. A higher fraction than $20 \%$ FVC was not conducted because, for some subjects, the breathing flows were too high, which caused fluctuation in the sampling flow of CPC.

Lung deposition measurements were normally conducted through the mouth route, but not the nose. This was because of less aerosol deposition in the mouth and more consistent anatomical dimensions of the mouth-laryngeal channel. On the other hand, the variations in nostril hairs, mucus layer in the nasal cavity, and the face-seal of the respirator all made the nasal route measurements more challenging. In Fig. 7, both local deposition efficiency curves (monodisperse $1 \mu \mathrm{m}$ aerosol particles and breathing frequency of 15 breaths $\mathrm{min}^{-1}$ ) through the mouth and nose were shown and compared. The nasal route showed higher deposition efficiency in the early stage of penetration volume of $200 \mathrm{~mL}$, likely due to the complex configuration of the nasal turbinate. The deposition efficiency curves downstream of the trachea (beyond $200 \mathrm{~mL}$ ) were almost identical. This is because the same subject performed the same breathing maneuver, almost at the same time.

\section{CONCLUSIONS}

The regional lung deposition data obtained using the newly developed rapid measurement system displayed good agreement with previous studies based on the bolus technique. The difference in the total lung deposition between Taiwanese and Caucasian subjects was found to be negligible. The total lung deposition curve, as a function of particle size, showed that the deposition efficiency of particles smaller than $0.3 \mu \mathrm{m}$ increased with decreasing particle size, whereas that of particles larger than $0.3 \mu \mathrm{m}$ 


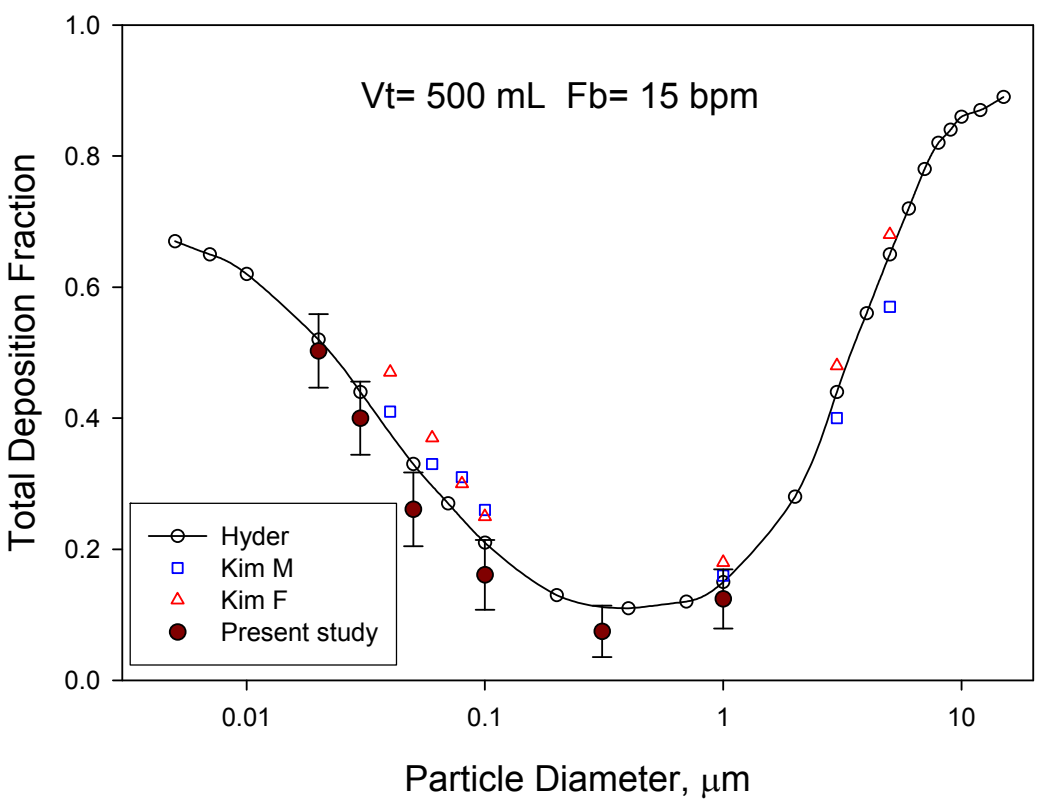

Fig. 5. Total deposition fraction compared with Kim's \& Hyder's data.

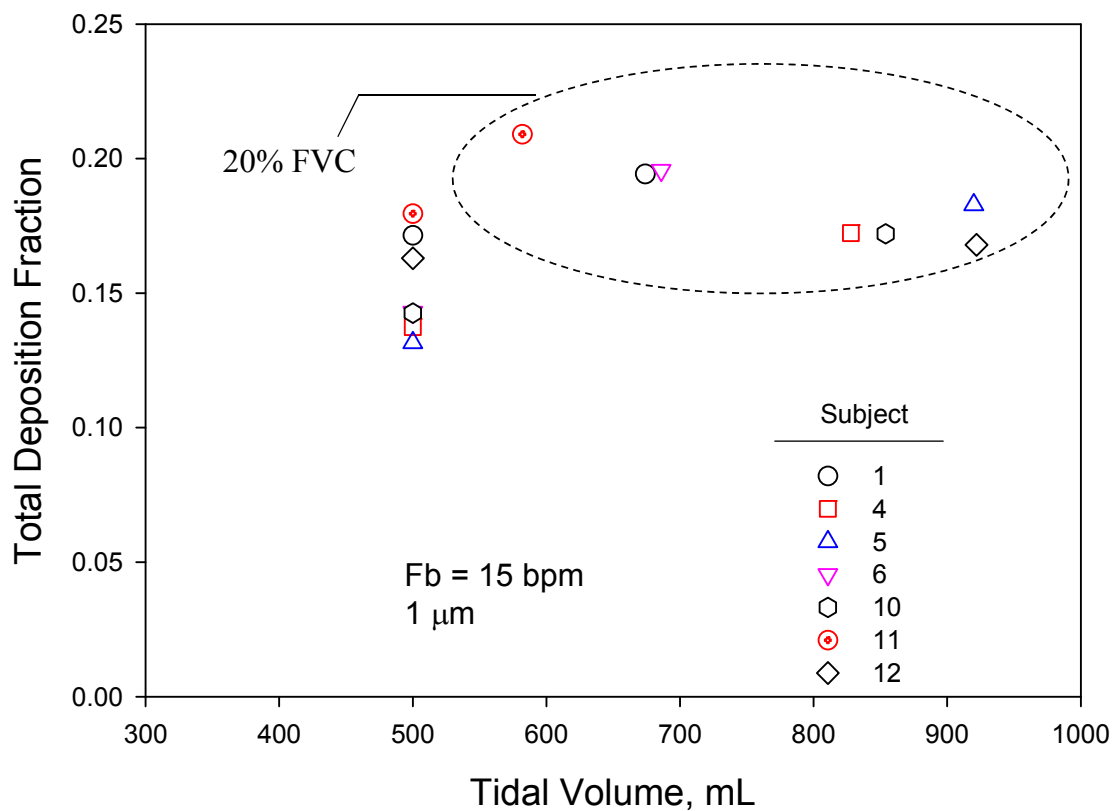

Fig. 6. Comparison of total deposition fraction at fixed $500 \mathrm{~mL}$ and $20 \% \mathrm{FVC}$ as tidal volume.

increased with the particle size. This difference is due to the fact that small particles tend to collect through diffusion, while large particles tend to collect through inertial impaction. The collection minimum appeared at around $0.3 \mu \mathrm{m}$, indicating that human lungs possess the same filtration characteristics as a mechanical filter.

The local deposition efficiency of Taiwanese lungs was higher than that found in Kim's research, which may be attributable to the use of (1) different measurement methods (the rapid measurement system in the present study vs. the bolus technique in the previous one) and (2) different breathing patterns (a sinusoidal wave in the present study vs. a constant flow in the previous one). The difference in local deposition efficiency was especially noticeable in the deep lung region $(400-500 \mathrm{~mL})$. We speculate that this phenomenon was due to dilution by relatively clean air in the functional residual capacity, but more studies are needed for validation.

The individual trend in local deposition efficiency was quite consistent for each subject but varied with time. The variation between subjects, however, was significant, even for subjects with approximately the same lung functionality. For all subjects, the total deposition fraction increased with the tidal volume, as aerosol particles had to travel a greater distance and flow over more surface area, but the total deposition varied even with a fixed tidal volume due to the 


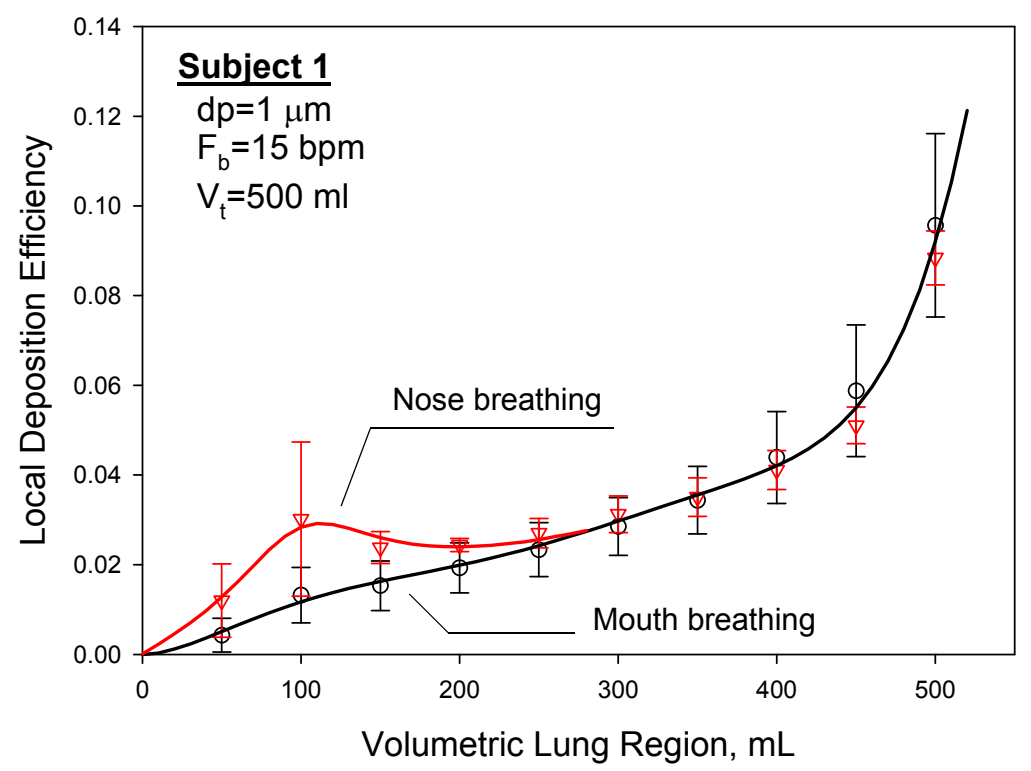

Fig. 7. Comparison of local deposition efficiency measured by nose and mouth breathing.

differences in lung functionality between participants. Furthermore, when a fixed fraction of the FVC was employed to replace the fixed tidal volume, the total deposition fraction became less dependent on the tidal volume, indicating the deposition efficiency during normal breathing is about the same for all subjects. Nevertheless, a person with a higher tidal volume collects a greater amount of aerosol deposits. The local deposition efficiency of the nasal route was higher than that of the oral route in the early stage of penetration $(200 \mathrm{~mL})$. The deposition efficiency curves downstream of the trachea (beyond $200 \mathrm{~mL}$ ) were almost identical for both nasal and oral breathing.

\section{ACKNOWLEDGMENTS}

The authors would like to thank the Ministry of Science and Technology of Taiwan (Grant No. MOST 105-3011-F002-008) for the financial supports.

\section{REFERENCES}

Ali, M., Mazumder, M.K. and Martonen, T.B. (2009). Measurements of electrodynamic effects on the deposition of MDI and DPI aerosols in a replica cast of human oral-pharyngeal-laryngeal airways. J. Aerosol Med. Pulm. Drug Deliv. 22: 35-44.

Ali, M., Reddy, R.N. and Mazumder, M.K. (2008). Electrostatic charge effect on respirable aerosol particle deposition in a cadaver based throat cast replica. $J$. Electrostat. 66: 401-406.

Brand, P., Rieger, C., Schulz, H., Beinert, T. and Heyder, J. (1997). Aerosol bolus dispersion in healthy subjects. Eur. Respir. J. 10: 460-467.

Chan, T.L. and Lippmann, M. (1980). Experimental measurements and empirical modelling of the regional deposition of inhaled particles in humans. Am. Ind. Hyg. Assoc. J. 41: 399-409.
Chang, K.N., Huang, S.H., Chen, C.W., Wu, H.D., Chen, Y.K., Lai, C.Y. and Chen, C.C. (2013). A sampling train for rapid measurement of regional lung deposition. Aerosol Air Qual. Res. 13: 608-617.

Cohen, B.S., Xiong, J.Q., Fang, C.P. and Li, W. (1998). Deposition of charged particles on lung airways. Health Phys. 74: 554-560.

Darquenne, C. (2012). Aerosol Deposition in Health and Disease. J. Aerosol Med. Pulm. Drug Deliv. 25: 140147.

Grgic, B., Martin, A.R. and Finlay, W.H. (2006). The effect of unsteady flow rate increase on in vitro mouththroat deposition of inhaled boluses. J. Aerosol Sci. 37: $1222-1233$.

Hashish, A.H., Fleming, J.S., Conway, J., Halson, P., Moore, E., Williams, T.J., Bailey, A.G., Nassim, M. and Holgate, S.T. (1998). Lung deposition of particles by airway generation in healthy subjects: Three-dimensional radionuclide imaging and numerical model prediction. $J$. Aerosol Sci. 29: 205-215.

Heyder, J., Gebhart, J., Rudolf, G., Schiller, C.F. and Stahlhofen, W. (1986). Deposition of particles in the human respiratory tract in the size range $0.005-15 \mu \mathrm{m}$. J. Aerosol Sci. 17: 811-825.

Heyder, J., Blanchard, J.D., Feldman, H.A. and Brain, J.D. (1988). Convective mixing in human respiratory tract: Estimates with aerosol boli. J. Appl. Physiol. 64: 12731278.

Inthavong, K., Choi, L.T., Tu, J., Ding, S. and Thien, F. (2010). Micron particle deposition in a tracheobronchial airway model under different breathing conditions. Med. Eng. Phys. 32: 1198-1212.

Jaques, P.A. and Kim, C.S. (2000). Measurement of total lung deposition of inhaled ultrafine particles in healthy men and women. Inhalation Toxicol. 12: 715-731.

Kim, C.S., Hu, S.C., DeWitt, P. and Gerrity, T.R. (1996). Assessment of regional deposition of inhaled particles in 
human lungs by serial bolus delivery method. J. Appl. Physiol. 81: 2203-2213.

Kim, C.S. and Jaques, P.A. (2005). Total lung deposition of ultrafine particles in elderly subjects during controlled breathing. Inhalation Toxicol. 17: 387-399.

Kim, C.S. and $\mathrm{Hu}$, S.C. (2006). Total respiratory tract deposition of fine micrometer-sized particles in healthy adults: Empirical equations for sex and breathing pattern. J. Appl. Physiol. 101: 401-412.

Lippmann, M. and Albert, R.E. (1969). The effect of particle size on the regional deposition of inhaled aerosols in the human respiratory tract. Am. Ind. Hyg. Assoc. J. 30: 257-275.

Möller, W., Felten, K., Seitz, J., Sommerer, K., Takenaka, S., Wiebert, P., Philipson, K., Svartengren, M. and Kreyling, W.G. (2006). A generator for the production of radiolabelled ultrafine carbonaceous particles for deposition and clearance studies in the respiratory tract J. Aerosol Sci. 37: 631-644.

Patterson, R.F., Zhang, Q., Zheng, M. and Zhu, Y. (2014). Particle deposition in respiratory tracts of school-aged children. Aerosol Air Qual. Res. 14: 64-73.

Rissler, J., Nicklasson, H., Gudmundsson, A., Wollmer, P.,
Swietlicki, E. and Löndahl, J. (2017). A set-up for respiratory tract deposition efficiency measurements $(15-5000 \mathrm{~nm})$ and first results for a group of children and adults. Aerosol Air Qual. Res. 17: 1244-1255.

Stahlhofen, W., Gebhart, J. and Heyder, J. (1980). Experimental determination of the regional deposition of aerosol particles in the human respiratory tract. $\mathrm{Am}$. Ind. Hyg. Assoc. J. 41: 385-398.

Sturm, R. and Hofmann, W. (2009). A theoretical approach to the deposition and clearance of fibers with variable size in the human respiratory tract. J. Hazard. Mater. 170: 210-218.

$\mathrm{Su}$, W.C. and Cheng, Y.S. (2006). Deposition of fiber in a human airway replica. J. Aerosol Sci. 37: 1429-1441.

Zhou, Y., Sun, J. and Cheng, Y.S. (2011). Comparison of deposition in the USP and physical mouth-throat models with solid and liquid particles. J. Aerosol Med. Pulm. Drug Deliv. 24: 277-284.

Received for review, June 13, 2018

Revised, August 1, 2018 Accepted, October 10, 2018 